\title{
Finite Population Distribution Function Estimation Using Auxiliary Information Under Simple Random Sampling
}

\author{
Sohaib Ahmad ${ }^{1}$, Sardar Hussain ${ }^{2}$, and Sohail Ahmad ${ }^{* 2}$ \\ ${ }^{1}$ Department of Statistics Abdul Wali Khan University Mardan Pakistan \\ ${ }^{2}$ Department of Statistics, Quaid-I-azam University, Islamabad, Pakistan \\ *Corresponding Author: asohail@stat.qau.edu.pk
}

\begin{abstract}
In this paper, a new estimator for estimating the finite population distribution function(DF) are propose using supplementary information on the DF of the auxiliary variable under simple random sampling. A comparative study is conducted to compare, theoretically and numerically, the adapted distribution function estimators of Cochran (1940), Murthy (1967), Bahl and Tuteja (1991), Rao (1991), Singh et al. (2009) and Grover and Kaur (2014) with the proposed estimators. It is found that the proposed estimators always perform better than the adapted estimators in terms of MSE and percentage relative efficiency.
\end{abstract}

Keywords: Distribution Function, Estimator, Sampling distribution, Statistics.

\section{Introduction}

In the theory of survey sampling, suitable use of the auxiliary information plays an important role in increasing the precision of an estimator of the unknown population parameter(s). A lot of ratio, product and regression type estimators of the population mean have been proposed in the literature of survey sampling. Ratio estimators are widely used to estimate the population mean when there exists a high correlation between the study variable and the auxiliary variable. Several authors including Murthy (1967), Srivastava and Jhajj (1983), Rao (1991), Upadhyaya and Singh (1999), Singh (2003), Shabbir and Gupta (2005), Kadilar and Cingi (2005), Kadilar and Cingi (2006) Gupta and Shabbir (2008), Singh et al. (2009) Grover and Kaur (2011) and Grover and Kaur (2014), have devised different types of estimators that utilize supplementary information in terms of one or more auxiliary variables to estimate the population mean.

The rest of paper is organized as follows. In Section 2, some notations are given. In Section 3, we adapt some estimators of finite population mean for the finite (DF). The proposed estimators are given in Section 4. In Section 5, numerical comparisons are performed. And 6 concern with empirical studies, finally, conclusions are drawn in Section 7. 


\section{Notations}

Consider a finite population $\Omega=\{1,2, \ldots, N\}$ of $N$ distinct units. In order to estimate the finite population distribution function, a sample of size $n$ units is drawn from this population using simple random sampling without replacement. Let

$Y$ : the study variable,

$X$ : the auxiliary variable,

$Z$ : ranks of $X$,

$I\left(Y \leq t_{y}\right)$ : indicator variable based on $Y$,

$I\left(X \leq t_{x}\right)$ : indicator variable based on $X$,

where $x=\tilde{X}$ and $y=\tilde{Y}$. Here, $\tilde{X}$ and $\tilde{Y}$ is the population medians of $X$ and $Y$

$F\left(t_{y}\right)=\sum_{i=1}^{N} I\left(Y_{i} \leq t_{y}\right) / N$ : the population distribution function of $Y$,

$\hat{F}\left(t_{y}\right)=\sum_{i=1}^{n} I\left(Y_{i} \leq t_{y}\right) / n$ : the sample distribution function of $Y$,

$F\left(t_{x}\right)=\sum_{i=1}^{N} I\left(X_{i} \leq t_{x}\right) / N$ : the population distribution function of $X$,

$\hat{F}\left(t_{x}\right)=\sum_{i=1}^{n} I\left(X_{i} \leq t_{x}\right) / n$ : the sample distribution function of $X$,

$S_{F_{t_{y}}}^{2}=\sum_{i=1}^{N}\left(I\left(Y_{i} \leq t_{y}\right)-F\left(t_{y}\right)\right)^{2} /(N-1)$ : the population variance of $I\left(Y \leq t_{y}\right)$,

$S_{F_{t_{x}}}^{2}=\sum_{i=1}^{N}\left(I\left(X_{i} \leq t_{x}\right)-F\left(t_{x}\right)\right)^{2} /(N-1)$ : the population variance of $I\left(X \leq t_{x}\right)$,

$C_{F_{t_{y}}}=S_{F_{t_{y}}} / F\left(t_{y}\right)$ : the population coefficient of variation of $I\left(Y \leq t_{y}\right)$,

$C_{F_{t_{x}}}=S_{F_{t_{x}}} / F\left(t_{x}\right)$ : the population coefficient of variation of $I\left(X \leq t_{x}\right)$,

$S_{F_{t_{y}} F_{t_{x}}}=\sum_{i=1}^{N}\left\{\left(I\left(Y_{i} \leq t_{y}\right)-F\left(t_{y}\right)\right)\left(I\left(X_{i} \leq t_{x}\right)-F\left(t_{x}\right)\right)\right\} /(N-1)$ : the population covariance between $I\left(Y \leq t_{y}\right)$ and $I\left(X \leq t_{x}\right)$,

$R_{F_{t_{y}} F_{t_{x}}}=S_{F_{t_{y}} F_{t_{x}}} /\left(S_{F_{t_{y}}} S_{F_{t_{x}}}\right)$ : the population correlation coefficient between $I\left(Y \leq t_{y}\right)$ and $I\left(X \leq t_{x}\right)$,

In order to obtain the biases and mean squared errors (MSEs) of the existing and proposed estimators of $F\left(t_{y}\right)$, we consider the following relative error terms. Let

$$
e_{0}=\frac{\hat{F}\left(t_{y}\right)-F\left(t_{y}\right)}{F\left(t_{y}\right)} \text { and } e_{1}=\frac{\hat{F}\left(t_{x}\right)-F\left(t_{x}\right)}{F\left(t_{x}\right)},
$$

such that $e_{i}=0$ for $i=0,1$, where $E(\cdot)$ is the mathematical expectation of $(\cdot)$.

$$
E\left(e_{0}^{2}\right)=\lambda C_{F_{t_{y}}}^{2}=\varphi_{20}, E\left(e_{1}^{2}\right)=\lambda C_{F_{t_{x}}}^{2}=\varphi_{02}, E\left(e_{0} e_{1}\right)=\lambda R_{F_{t_{y}} F_{t_{x}}} C_{F_{t_{y}}} C_{F_{t_{x}}}=\varphi_{11} .
$$

\section{Adapted estimators in simple random sampling}

In this section, some estimators of finite population mean are adapted for estimating the finite CDF under simple random sampling, which are exist for mean estimation. The biases and MSEs of these adapted estimators are derived under the first order of approximation.

1. The traditional unbiased estimator of $F\left(t_{y}\right)$ is

$$
\hat{F}\left(t_{y}\right)=\frac{1}{n} \sum_{i=1}^{n} I\left(Y_{i} \leq t_{y}\right)
$$

The variance of $\hat{F}\left(t_{y}\right)$ is 
Finite Population Distribution Function Estimation Using Auxiliary Information Under Simple Random Sampling

$$
\operatorname{Var}\left(\widehat{F}\left(t_{y}\right)\right)=F^{2}\left(t_{y}\right) \varphi_{20} .
$$

2. Cochran (1940) existing ratio estimator of $F\left(t_{y}\right)$ is

$$
\hat{F}_{R}\left(t_{y}\right)=\hat{F}\left(t_{y}\right)\left(\frac{F\left(t_{x}\right)}{\hat{F}\left(t_{x}\right)}\right) \text {. }
$$

The bias and MSE of $\hat{F}_{R}\left(t_{y}\right)$, to the first order of approximation, are

$$
\begin{aligned}
& \operatorname{Bias}\left(\widehat{F}_{R}\left(t_{y}\right)\right) \cong F\left(t_{y}\right)\left(\varphi_{02}-\varphi_{11}\right), \\
& \operatorname{MSE}\left(\hat{F}_{R}\left(t_{y}\right)\right) \cong F^{2}\left(t_{y}\right)\left(\varphi_{20}+\varphi_{02}-2 \varphi_{11}\right) .
\end{aligned}
$$

3. Murthy (1964) existing product estimator of $F\left(t_{y}\right)$ is

$$
\hat{F}_{P}\left(t_{y}\right)=\hat{F}\left(t_{y}\right)\left(\frac{\hat{F}\left(t_{x}\right)}{F\left(t_{x}\right)}\right) \text {. }
$$

The bias and MSE of $\hat{F}_{P}\left(t_{y}\right)$, to the first order of approximation, are

$$
\begin{aligned}
& \operatorname{Bias}\left(\hat{F}_{P}\left(t_{y}\right)\right)=F\left(t_{y}\right) \varphi_{11}, \\
& \operatorname{MSE}\left(\hat{F}_{P}\left(t_{y}\right)\right) \cong F^{2}\left(t_{y}\right)\left(\varphi_{20}+\varphi_{02}+2 \varphi_{11}\right)
\end{aligned}
$$

4. Following Bahl and Tuteja (1991), the combined ratio-type and product-type exponential estimators of $F\left(t_{y}\right)$, respectively, are

$$
\begin{aligned}
& \hat{F}_{B T, R}\left(t_{y}\right)=\hat{F}\left(t_{y}\right) \exp \left(\frac{F\left(t_{x}\right)-\hat{F}\left(t_{x}\right)}{\hat{F}\left(t_{x}\right)+F\left(t_{x}\right)}\right), \\
& \hat{F}_{B T, P}\left(t_{y}\right)=\hat{F}\left(t_{y}\right) \exp \left(\frac{\hat{F}\left(t_{x}\right)-F\left(t_{x}\right)}{\hat{F}\left(t_{x}\right)+F\left(t_{x}\right)}\right) .
\end{aligned}
$$

The biases and MSEs of $\hat{F}_{B T, R}\left(t_{y}\right)$ and $\widehat{F}_{B T, P}\left(t_{y}\right)$, to the first order of approximation, are

$$
\begin{aligned}
& \operatorname{Bias}\left(\widehat{F}_{B T, R}\left(t_{y}\right)\right) \cong F\left(t_{y}\right)\left(\frac{3}{8} \varphi_{020}-\frac{1}{2} \varphi_{110}\right), \\
& \operatorname{MSE}\left(\widehat{F}_{B T, R}\left(t_{y}\right)\right) \cong \frac{F^{2}\left(t_{y}\right)}{4}\left(4 \varphi_{200}+\varphi_{020}-4 \varphi_{110}\right),
\end{aligned}
$$

and

$$
\begin{aligned}
& \operatorname{Bias}\left(\hat{F}_{B T, P}\left(t_{y}\right)\right) \cong F\left(t_{y}\right)\left(\frac{1}{2} \varphi_{110}-\frac{1}{8} \varphi_{020}\right), \\
& \operatorname{MSE}\left(\hat{F}_{B T, P}\left(t_{y}\right)\right) \cong \frac{F^{2}\left(t_{y}\right)}{4}\left(4 \varphi_{200}+\varphi_{020}+4 \varphi_{110}\right),
\end{aligned}
$$

respectively.

5. The existing regression estimator of $F\left(t_{y}\right)$ is

$$
\hat{F}_{R e g}\left(t_{y}\right)=\hat{F}\left(t_{y}\right)+m\left(F\left(t_{x}\right)-\hat{F}\left(t_{x}\right)\right),
$$

where $m$ is an unknown constant. Here, $\hat{F}_{R e g}\left(t_{y}\right)$ is an unbiased estimator of $\hat{F}\left(t_{y}\right)$. The minimum variance of $\hat{F}_{R e g}\left(t_{y}\right)$ at the optimum value $m_{(\mathrm{opt})}=\left(F\left(t_{y}\right) \varphi_{11}\right) /\left(F\left(t_{x}\right) \varphi_{02}\right)$ is

$$
\operatorname{Var}_{\min }\left(\hat{F}_{R e g}\left(t_{y}\right)\right)=\frac{F^{2}\left(t_{y}\right)\left(\varphi_{20} \varphi_{02}-\varphi_{11}^{2}\right)}{\varphi_{02}}
$$

Here, (12) may be written as

$$
\operatorname{Var}_{\min }\left(\widehat{F}_{R e g}\left(t_{y}\right)\right)=F^{2}\left(t_{y}\right) \varphi_{20}\left(1-\rho_{12}^{2}\right)
$$

6. Rao (1991) existing difference-type estimator of $F\left(t_{y}\right)$ is

$$
\widehat{F}_{R, D}\left(t_{y}\right)=m_{1} \hat{F}\left(t_{y}\right)+m_{2}\left(F\left(t_{x}\right)-\hat{F}\left(t_{x}\right)\right),
$$


where $m_{1}$ and $m_{2}$ are unknown constants. The bias and MSE of $\widehat{F}_{R, D}\left(t_{y}\right)$, to the first order of approximation, are

$$
\begin{aligned}
\operatorname{Bias}\left(\hat{F}_{R, D}\left(t_{y}\right)\right)= & F\left(t_{y}\right)\left(m_{1}-1\right) \\
\operatorname{MSE}\left(\hat{F}_{R, D}\left(t_{y}\right)\right) \cong & F^{2}\left(t_{y}\right)-2 m_{1} F^{2}\left(t_{y}\right)+m_{1}^{2} F^{2}\left(t_{y}\right)+m_{1}^{2} F^{2}\left(t_{y}\right) \varphi_{20} \\
& -2 m_{1} m_{2} F\left(t_{y}\right) F\left(t_{x}\right) \varphi_{11}+m_{2}^{2} F^{2}\left(t_{x}\right) \varphi_{02} .
\end{aligned}
$$

The optimum values of $m_{1}$ and $m_{2}$, determined by minimizing (15), are

$$
\begin{aligned}
& m_{1(\mathrm{opt})}=\frac{\varphi_{02}}{\left(\varphi_{02} \varphi_{20}-\varphi_{11}^{2}+\varphi_{02}\right)}, \\
& m_{2 \text { (opt) }}=\frac{F\left(t_{y}\right) \varphi_{11}}{F\left(t_{x}\right)\left(\varphi_{20} \varphi_{02}-\varphi_{11}^{2}+\varphi_{02}\right)} .
\end{aligned}
$$

The minimum MSE of $\widehat{F}_{R, D}\left(t_{y}\right)$ at the optimum values of $m_{1}$ and $m_{2}$ is

$$
\operatorname{MSE}_{\min }\left(\widehat{F}_{R, D}\left(t_{y}\right)\right)=\frac{F^{2}\left(t_{y}\right)\left(\varphi_{20} \varphi_{02}-\varphi_{11}^{2}\right)}{\left(\varphi_{20} \varphi_{02}-\varphi_{11}^{2}+\varphi_{02}\right)}
$$

Here, (16) may be written as

$$
\operatorname{MSE}_{\min }\left(\widehat{F}_{R, D}\left(t_{y}\right)\right)=\frac{F^{2}\left(t_{y}\right) \varphi_{20}\left(1-\rho_{12}^{2}\right)}{1+\varphi_{20}\left(1-\rho_{12}^{2}\right)} .
$$

7. Singh et al. (2009) adapted generalized ratio-type exponential estimator of $F\left(t_{y}\right)$ is

$$
\hat{F}_{S}\left(t_{y}\right)=\hat{F}\left(t_{y}\right) \exp \left(\frac{a\left(F\left(t_{x}\right)-\hat{F}\left(t_{x}\right)\right)}{a\left(F\left(t_{x}\right)+\hat{F}\left(t_{x}\right)\right)+2 b}\right)
$$

where $a=1$ and $b=0$ are known constants. The bias and MSE of $\hat{F}_{S}\left(t_{y}\right)$, to the first order of approximation, are

$$
\begin{aligned}
& \operatorname{Bias}\left(\hat{F}_{S}\left(t_{y}\right)\right) \cong F\left(t_{y}\right)\left(\frac{3}{8} \theta^{2} \varphi_{02}-\frac{1}{2} \theta \varphi_{11}\right), \\
& \operatorname{MSE}\left(\hat{F}_{S}\left(t_{y}\right)\right) \cong \frac{F^{2}\left(t_{y}\right)}{4}\left(4 \varphi_{20}+\theta^{2} \varphi_{02}-4 \theta \varphi_{11}\right),
\end{aligned}
$$

where $\theta=a F\left(t_{x}\right) /\left(a F\left(t_{x}\right)+b\right)$. Here the value of $\theta$ become 1

8. Grover and Kaur (2014) adapted generalized class of ratio-type exponential estimator of $F\left(t_{y}\right)$ is

$$
\hat{F}_{G, K}\left(t_{y}\right)=\left\{m_{3} \hat{F}\left(t_{y}\right)+m_{4}\left(F\left(t_{x}\right)-\hat{F}\left(t_{x}\right)\right)\right\} \exp \left(\frac{a\left(F\left(t_{x}\right)-\hat{F}\left(t_{x}\right)\right)}{a\left(F\left(t_{x}\right)+\hat{F}\left(t_{x}\right)\right)+2 b}\right),
$$

where $m_{3}$ and $m_{4}$ are unknown constants. The bias and MSE of $\hat{F}_{G, K}\left(t_{y}\right)$, to the first order of approximation, are

$$
\begin{aligned}
\operatorname{Bias}\left(\hat{F}_{G, K}\left(t_{y}\right)\right) \cong F( & \left.t_{y}\right)\left(m_{3}-1\right)+\frac{3}{8} \theta^{2} m_{3} F\left(t_{y}\right)+\frac{1}{2} \theta m_{4} F\left(t_{x}\right) \varphi_{02}-\frac{1}{2} \theta F\left(t_{y}\right) \varphi_{11}, \\
\operatorname{MSE}\left(\hat{F}_{G, K}\left(t_{y}\right)\right) \cong & m_{4}^{2} F^{2}\left(t_{x}\right) \varphi_{02}+m_{3}^{2} F^{2}\left(t_{y}\right) \varphi_{20}+2 \theta m_{3} m_{4} F\left(t_{y}\right) F\left(t_{x}\right) \varphi_{02} \\
& -2 m_{3} m_{4} F\left(t_{y}\right) F\left(t_{x}\right) \varphi_{11}+F^{2}\left(t_{y}\right)-2 m_{3} F^{2}\left(t_{y}\right)+\theta m_{3}^{2} F^{2}\left(t_{y}\right) \\
& +m_{3} F^{2}\left(t_{y}\right) \varphi_{11}-\theta m_{4} F\left(t_{y}\right) F\left(t_{x}\right) \varphi_{02}-2 \theta m_{3}^{2} F^{2}\left(t_{y}\right) \varphi_{11} \\
& -\frac{3}{4} \theta^{2} m_{3} F^{2}\left(t_{y}\right) \varphi_{02}+\theta^{2} m_{3}^{2} F^{2}\left(t_{y}\right) \varphi_{02} .
\end{aligned}
$$

The optimum values of $m_{3}$ and $m_{4}$, determined by minimizing (21), are

$$
k_{3(\mathrm{opt})}=\frac{\varphi_{02}\left(\theta^{2} \varphi_{02}-8\right)}{8\left(-\varphi_{20} \varphi_{02}+\varphi_{11}^{2}-\varphi_{02}\right)},
$$


Finite Population Distribution Function Estimation Using Auxiliary Information Under Simple Random Sampling

$$
k_{4(\mathrm{opt})}=\frac{F\left(t_{y}\right)\left(\theta^{3} \varphi_{02}^{2}-\theta^{2} \varphi_{02} \varphi_{11}+4 \theta \varphi_{20} \varphi_{02}-4 \theta \varphi_{11}^{2}-4 \theta \varphi_{02}+8 \varphi_{11}\right)}{8 F\left(t_{x}\right)\left(\varphi_{20} \varphi_{02}-\varphi_{11}^{2}+\varphi_{02}\right)} .
$$

The simplified minimum MSE of $\hat{F}_{G, K}\left(t_{y}\right)$ at the optimum values of $m_{3}$ and $m_{4}$ is

$$
\operatorname{MSE}_{\min }\left(\widehat{F}_{G, K}\left(t_{y}\right)\right) \cong \frac{F^{2}\left(t_{y}\right)}{64}\left(64-16 \theta^{2} \varphi_{02}-\frac{\varphi_{02}\left(-8+\theta^{2} \varphi_{02}\right)^{2}}{\varphi_{02}\left(1+\varphi_{20}\right)-\varphi_{11}^{2}}\right) \text {. }
$$

\section{Proposed estimator in simple random sampling}

On the lines of $\hat{F}_{R, D}\left(t_{y}\right), \hat{F}_{S}\left(t_{y}\right)$ and average of $\hat{F}_{B T, R}\left(t_{y}\right)$ and $\hat{F}_{B T, P}\left(t_{y}\right)$, a new estimator is proposed for estimating $F\left(t_{y}\right)$ is given by

$$
\hat{F}_{\text {Prop }}\left(t_{y}\right)=\left[\begin{array}{c}
\frac{\hat{F}\left(t_{y}\right)}{2}\left\{\exp \left(\frac{F\left(t_{x}\right)-\hat{F}\left(t_{x}\right)}{\hat{F}\left(t_{x}\right)+F\left(t_{x}\right)}\right)+\exp \left(\frac{\hat{F}\left(t_{x}\right)-F\left(t_{x}\right)}{\hat{F}\left(t_{x}\right)+F\left(t_{x}\right)}\right)\right\} \\
+m_{5}\left(F\left(t_{x}\right)-\hat{F}\left(t_{x}\right)\right)+m_{6} \hat{F}\left(t_{y}\right)
\end{array}\right] \exp \left(\frac{F\left(t_{x}\right)-\hat{F}\left(t_{x}\right)}{F\left(t_{x}\right)+\hat{F}\left(t_{x}\right)}\right),
$$

where $m_{5}$ and $m_{6}$, are determined late. The estimator $\hat{F}_{\text {Prop }}\left(t_{y}\right)$ can also be written as

$\widehat{F}_{\text {Prop }}\left(t_{y}\right)=\left\{F\left(t_{y}\right)\left(1+e_{0}\right)\left(1+m_{6}\right)-m_{5} e_{1} F\left(t_{x}\right)+\frac{1}{8} F\left(t_{y}\right)\right\}\left(1-\frac{1}{2} e_{1}+\frac{3}{8} \xi_{1}^{2}+\cdots\right)$.

Simplifying (23) and keeping terms only up to the second power of $\xi$ 's, we can write

$$
\begin{aligned}
\left(\hat{F}_{\text {Prop }}\left(t_{y}\right)-F\left(t_{y}\right)\right) \cong & m_{6} F\left(t_{y}\right)+F\left(t_{y}\right) e_{0}+m_{6} F\left(t_{y}\right) e_{0}-\frac{1}{2} F\left(t_{y}\right) e_{1}+\frac{1}{2} m_{6} F\left(t_{y}\right) e_{1} \\
& -\frac{1}{2} F\left(t_{y}\right) e_{0} e_{1}+m_{5} F\left(t_{y}\right) e_{1}^{2}+\frac{1}{2} m_{5} F\left(t_{x}\right) e_{1}^{2}-\frac{1}{2} m_{6} F\left(t_{y}\right) e_{0} e_{1} \\
& +\frac{3}{8} m_{6} F\left(t_{y}\right) e_{1}^{2}-m_{5} F\left(t_{x}\right) e_{1} .
\end{aligned}
$$

The bias and MSE of $\hat{F}_{\text {Prop }}\left(t_{y}\right)$, to the first order of approximation,respectively, are

$$
\begin{aligned}
\operatorname{Bias}\left(\widehat{F}_{\text {Prop }}\left(t_{y}\right)\right) \cong & \frac{1}{2} F\left(t_{y}\right) \varphi_{02}-\frac{1}{2} F\left(t_{y}\right) \varphi_{11}+\frac{1}{2} m_{5} \varphi_{02}+m_{6} F\left(t_{y}\right) \\
& +\frac{3}{8} m_{6}^{2} F\left(t_{y}\right) \varphi_{02}-\frac{1}{2} m_{6} F\left(t_{y}\right) \varphi_{11}, \\
\operatorname{MSE}\left(\hat{F}_{\text {Prop }}\left(t_{y}\right)\right) \cong & \frac{1}{4} \varphi_{02}\left\{\left(F\left(t_{y}\right)+2 m_{5} F\left(t_{x}\right)\right)^{2}-2 F\left(t_{y}\right)\left(3 F\left(t_{y}\right)+4 m_{5} F\left(t_{x}\right)\right) m_{6}+\right. \\
& \left.4 m_{6}^{2} F^{2}\left(t_{y}\right)\right\}-F\left(t_{y}\right) \varphi_{11}\left(1+m_{6}\right)\left(F\left(t_{y}\right)+2 m_{5} F\left(t_{x}\right)+2 m_{6} F\left(t_{y}\right)\right) \\
& +F^{2}\left\{m_{6}^{2}+\varphi_{20}\left(1+m_{6}\right)^{2}\right\} .
\end{aligned}
$$

The optimum values of $m_{5}, m_{6}$ and $m_{7}$, determined by minimizing (25), are

$$
\begin{aligned}
& m_{5(\mathrm{opt})}=\frac{F\left(t_{y}\right)\left[-4 \frac{\varphi_{11}}{\varphi_{20}^{-1 / 2} \varphi_{02}^{1 / 2}}+\varphi_{02}^{1 / 2}\left\{2-\varphi_{02}+\varphi_{11}+2\left(-1+\frac{\varphi_{11}^{2}}{\varphi_{20} \varphi_{02}}\right) \varphi_{20}\right\}\right]}{4 F\left(t_{x}\right) \varphi_{02}^{1 / 2}\left\{-1+\left(-1+\frac{\varphi_{11}^{2}}{\varphi_{20} \varphi_{02}}\right)\right\}} \text { and } \\
& m_{6 \text { (opt) }}=\frac{\varphi_{02}-4\left(-1+\frac{\varphi_{11}^{2}}{\varphi_{20} \varphi_{02}}\right) \varphi_{20}}{4\left\{-1+\left(-1+\frac{\varphi_{11}^{2}}{\varphi_{20} \varphi_{02}}\right)\right\}}
\end{aligned}
$$

respectively. The simplified minimum MSE of $\hat{F}_{\text {Prop }}\left(t_{y}\right)$ at the optimum values of $m_{5}$ and $m_{6}$ is given by 


$$
\operatorname{MSE}_{\text {min }}\left(\hat{F}_{\text {Prop }}\left(t_{y}\right)\right) \cong \frac{F^{2}\left(t_{y}\right)\left\{16 \varphi_{02}^{2}-8\left(-1+\frac{\varphi_{11}^{2}}{\varphi_{20} \varphi_{02}}\right)\left(-2+\varphi_{02}\right) \varphi_{20}\right\}}{16\left\{-1+\left(-1+\frac{\varphi_{11}^{2}}{\varphi_{20} \varphi_{02}}\right)\right\}}
$$

\section{Efficiency comparisons in simple random sampling}

In this section, the adapted and proposed estimators of $F\left(t_{y}\right)$ are compared in terms of the minimum MSEs.

1. From (2) and (26),

$$
\begin{aligned}
& \operatorname{MSE}_{\text {min }}\left(\hat{F}_{\text {Prop }}\left(t_{y}\right)\right)<\operatorname{Var}\left(\hat{F}\left(t_{y}\right)\right) \text { if } \\
& \operatorname{Var}\left(\hat{F}\left(t_{y}\right)\right)-\operatorname{MSE}_{\text {min }}\left(\hat{F}_{\text {Prop }}\left(t_{y}\right)\right)>0 .
\end{aligned}
$$

2. From (4) and (26),

$$
\begin{aligned}
& \operatorname{MSE}_{\text {min }}\left(\hat{F}_{\text {Prop }}\left(t_{y}\right)\right)<\operatorname{MSE}\left(\widehat{F}_{R}\left(t_{y}\right)\right) \text { if } \\
& \operatorname{MSE}\left(\hat{F}_{R}\left(t_{y}\right)\right)-\operatorname{MSE}_{\text {min }}\left(\hat{F}_{\text {Prop }}\left(t_{y}\right)\right)>0 .
\end{aligned}
$$

3. From (6) and (26),

$$
\begin{aligned}
& \operatorname{MSE}_{\text {min }}\left(\hat{F}_{\text {Prop }}\left(t_{y}\right)\right)<\operatorname{MSE}\left(\hat{F}_{P}\left(t_{y}\right)\right) \text { if } \\
& \operatorname{MSE}\left(\hat{F}_{P}\left(t_{y}\right)\right)-\operatorname{MSE}_{\text {min }}\left(\widehat{F}_{\text {Prop }}\left(t_{y}\right)\right)>0 .
\end{aligned}
$$

4. From (10) and (26),

$$
\begin{aligned}
& \operatorname{MSE}_{\text {min }}\left(\widehat{F}_{\text {Prop }}\left(t_{y}\right)\right)<\operatorname{MSE}\left(\widehat{F}_{B T, R}\left(t_{y}\right)\right) \text { if } \\
& \operatorname{MSE}\left(\widehat{F}_{B T, R}\left(t_{y}\right)\right)-\operatorname{MSE}_{\text {min }}\left(\widehat{F}_{\text {Prop }}\left(t_{y}\right)\right)>0 .
\end{aligned}
$$

5. From (13) and (26),

$$
\begin{aligned}
& \operatorname{MSE}_{\text {min }}\left(\widehat{F}_{\text {Prop }}\left(t_{y}\right)\right)<\operatorname{MSE}\left(\widehat{F}_{B T, P}\left(t_{y}\right)\right) \text { if } \\
& \operatorname{MSE}\left(\widehat{F}_{B T, P}\left(t_{y}\right)\right)-\operatorname{MSE}_{\min }\left(\widehat{F}_{\text {Prop }}\left(t_{y}\right)\right)>0 .
\end{aligned}
$$

6. From (17) and (26),

$$
\begin{aligned}
& \operatorname{MSE}_{\text {min }}\left(\hat{F}_{\text {Prop }}\left(t_{y}\right)\right)<\operatorname{Var}_{\text {min }}\left(\hat{F}_{\text {Reg }}\left(t_{y}\right)\right) \text { if } \\
& \operatorname{Var}_{\text {min }}\left(\widehat{F}_{\text {Reg }}\left(t_{y}\right)\right)-\operatorname{MSE}_{\text {min }}\left(\widehat{F}_{\text {Prop }}\left(t_{y}\right)\right)>0 .
\end{aligned}
$$

7. From (19) and (26),

$$
\begin{aligned}
& \operatorname{MSE}_{\text {min }}\left(\widehat{F}_{\text {Prop }}\left(t_{y}\right)\right)<\operatorname{MSE}_{\text {min }}\left(\widehat{F}_{R, D}\left(t_{y}\right)\right) \text { if } \\
& \operatorname{MSE}_{\text {min }}\left(\widehat{F}_{R, D}\left(t_{y}\right)\right)-\operatorname{MSE}_{\text {min }}\left(\widehat{F}_{\text {Prop }}\left(t_{y}\right)\right)>0 .
\end{aligned}
$$

8. From (22) and (26),

$$
\begin{aligned}
& \operatorname{MSE}_{\text {min }}\left(\hat{F}_{\text {Prop }}\left(t_{y}\right)\right)<\operatorname{MSE}_{\text {min }}\left(\widehat{F}_{G, K}\left(t_{y}\right)\right) \text { if } \\
& \operatorname{MSE}_{\text {min }}\left(\hat{F}_{G, K}\left(t_{y}\right)\right)-\operatorname{MSE}_{\text {min }}\left(\hat{F}_{\text {Prop }}\left(t_{y}\right)\right)>0 .
\end{aligned}
$$




\section{Empirical study}

In this section, we conduct a numerical study to investigate the performances of the adapted and DF estimators. For this purpose, five populations are considered. The summary statistics of these populations are reported in Table 1. The percentage relative efficiency (PRE) of an estimator $\hat{F}_{i}\left(t_{y}\right)$ with respect to $\hat{F}_{S R S}\left(t_{y}\right)$ is

$$
\operatorname{PRE}\left(\hat{F}_{i}\left(t_{y}\right), \hat{F}_{S R S}\left(t_{y}\right)\right)=\frac{\operatorname{Var}\left(\hat{F}_{S R S}\left(t_{y}\right)\right)}{\operatorname{MSE}_{\min }\left(\hat{F}_{i}\left(t_{y}\right)\right)} \times 100,
$$

where $i=S R S, P, \ldots$, Prop.

The MSEs and PREs of distribution function estimators, computed from five populations, are given in Tables 2 and 3.

Population I [Source: Singh, 2003]

$Y$ : Duration of sleep (in minutes) and $X$ : Age of old persons.

Population II [Source: Gujarati, 2009]

$Y$ : The eggs produced in 1990 (millions) and $X$ : The price per dozen (cents) in 1990

Population III [Source: Murthy, 1967]

$Y$ : The output of the factory and $X$ : The number of workers

Population IV [Source: Sarndal, 1992a]

$Y$ : Population in 1983 (in million) and $X$ : Population in 1980 (in million)

Population V [Source: Koyuncu and Kadilar, 2009]

$Y:$ Number of teachers and $X$ : number of students

Table 1: Summary statistics for Populations I-V

\begin{tabular}{|c|c|c|c|c|c|}
\hline Population & I & II & III & IV & V \\
\hline$N$ & 30 & 50 & 80 & 120 & 923 \\
\hline$n$ & 5 & 5 & 10 & 20 & 180 \\
\hline$\lambda$ & 0.16667 & 0.18 & 0.0875 & 0.04167 & 0.00447 \\
\hline$Q_{2(y)}$ & 387 & 831 & 5105 & 9.25 & 171 \\
\hline$Q_{2(x)}$ & 66.5 & 75.35 & 148 & 8.6 & 4123 \\
\hline$F\left(t_{y}\right)$ & 0.5 & 0.5 & 0.5 & 0.5 & 0.50163 \\
\hline$C_{F t_{y}}$ & 1.01709 & 1.01015 & 1.00631 & 1.00419 & 0.99729 \\
\hline$F\left(t_{x}\right)$ & 0.5 & 0.5 & 0.5 & 0.5 & 0.50054 \\
\hline$C_{F t_{x}}$ & 1.01709 & 1.01015 & 1.00631 & 1.00419 & 0.99946 \\
\hline$R_{F t_{y} F t_{x}}$ & -0.73333 & -0.12000 & 0.95 & 0.96667 & 0.84616 \\
\hline$\beta_{2}$ & 1 & 1 & 1 & 1 & 1 \\
\hline
\end{tabular}


Table 2: MSEs using Populations I-V

\begin{tabular}{|c|c|c|c|c|c|}
\hline Estimators & Population-I & Population-II & Population-III & Population-IV & Population-V \\
\hline$\widehat{F}_{S R S}(y)$ & 0.0431035 & 0.0459184 & 0.0221519 & 0.0105042 & 0.0011192 \\
\hline$\widehat{F}_{R}(y)$ & 0.1494253 & 0.1028571 & 0.0022152 & 0.0007003 & 0.0003451 \\
\hline$\widehat{F}_{P}(y)$ & 0.0237504 & 0.0812919 & 0.0865339 & 0.0413609 & 0.0041361 \\
\hline$\widehat{F}_{B, R}(y)$ & 0.0854885 & 0.0629082 & 0.0066456 & 0.0029762 & 0.0004512 \\
\hline$\widehat{F}_{B, P}(y)$ & 0.0222701 & 0.0518878 & 0.0487342 & 0.0232843 & 0.0023494 \\
\hline$\widehat{F}_{R e g}(y)$ & 0.0199234 & 0.0452571 & 0.0021598 & 0.0006886 & 0.0003179 \\
\hline$\widehat{F}_{R, D}(y)$ & 0.0184528 & 0.0383201 & 0.0021413 & 0.0006867 & 0.0003175 \\
\hline$\widehat{F}_{G, K}(y)$ & 0.0175499 & 0.0364489 & 0.0020635 & 0.0006726 & 0.0003171 \\
\hline$\widehat{F}_{\text {Prop }}(y)$ & 0.0164319 & 0.0343546 & 0.0019248 & 0.0006448 & 0.0003165 \\
\hline
\end{tabular}

Table 3: PREs using Populations I-V

\begin{tabular}{|c|c|c|c|c|c|}
\hline Estimators & Population-I & Population-II & Population-III & Population-IV & Population-V \\
\hline$\widehat{F}_{S R S}(y)$ & 100.00 & 100.00 & 100.00 & 100.00 & 100.00 \\
\hline$\widehat{F}_{R}(y)$ & 28.85 & 44.64 & 1000.00 & 1500.00 & 324.29 \\
\hline$\hat{F}_{P}(y)$ & 181.49 & 56.49 & 25.60 & 25.40 & 27.06 \\
\hline$\widehat{F}_{B, R}(y)$ & 50.42 & 72.99 & 333.33 & 352.94 & 248.08 \\
\hline$\widehat{F}_{B, P}(y)$ & 193.55 & 88.50 & 45.45 & 45.11 & 47.64 \\
\hline$\widehat{F}_{R e g}(y)$ & 216.35 & 101.46 & 1025.64 & 1525.42 & 352.09 \\
\hline$\widehat{F}_{R, D}(y)$ & 233.59 & 119.83 & 1034.50 & 1529.63 & 352.53 \\
\hline$\widehat{F}_{G, K}(y)$ & 245.61 & 125.98 & 1073.53 & 1561.67 & 353.01 \\
\hline$\widehat{F}_{\text {Prop }}(y)$ & 262.32 & 133.66 & 1150.86 & 1629.11 & 353.67 \\
\hline
\end{tabular}

From the numerical results, presented in Tables 2 and 3, it is noted that the proposed estimator is more precise than the adapted distribution function estimators of f Cochran (1940), Murthy (1964), Bahl and Tuteja (1991), Rao (1991), Singh et al. (2009) and Grover and Kaur (2014), 15in terms of MSE and PRE.

\section{Conclusion}

In this paper, we have proposed a new estimator for estimating the finite population distribution function. The biases and MSEs of the proposed estimator were derived using first order approximation. Based on theoretical and numerical comparative studies, it has turned out that the proposed estimator is more precise than their existing counterparts.

\section{Aknowledgement}

We are thankful to the editor and management team for their schloarly guidence. 
Finite Population Distribution Function Estimation Using Auxiliary Information Under Simple Random Sampling

\section{References}

Bahl, S. and Tuteja, R. K. (1991). Ratio and product type exponential estimators. Journal of Information and Optimization Sciences, 12(1):159-164.

Cochran, W. (1940). The estimation of the yields of cereal experiments by sampling for the ratio of grain to total produce. The Journal of Agricultural Science, 30(2):262-275.

Grover, L. K. and Kaur, P. (2011). Ratio type exponential estimators of population mean under linear transformation of auxiliary variable: theory and methods. South African Statistical Journal,45(2):205-230.

Grover, L. K. and Kaur, P. (2014). A generalized class of ratio type exponential estimators of population mean under linear transformation of auxiliary variable. Communications in Statistics Simulation and Computation, 43(7):1552-1574.

Gujarati, D. N. (2009). Basic Econometrics. Tata McGraw-Hill Education.

Gupta, S. and Shabbir, J. (2008). On improvement in estimating the population mean in simple random sampling. Journal of Applied Statistics, 35(5):559-566.

Kadilar, C. and Cingi, H. (2005). A new ratio estimator in stratified random sampling. Communicationsin Statistics - Theory and Methods, 34(3):597-602.

Kadilar, C. and Cingi, H. (2006). Improvement in estimating the population mean in simple random sampling. Applied Mathematics Letters, 19(1):75-79.

Koyuncu, N. and Kadilar, C. (2009). Ratio and product estimators in stratified random sampling. Journal of Statistical Planning and Inference, 139(8):2552-2558.

Murthy, M. (1964). Product method of estimation. Sankhya: The Indian Journal of Statistics, Series A, 26(1):69-74.

Murthy, M. N. (1967). Sampling Theory and Methods. Statistical Pub. Society,Calcutta.

Rao, T. J. (1991). On certail methods of improving ration and regression estimators. Communicationsin Statistics-Theory and Methods, 20(10):3325-3340.

Sarndal, C. E. (1992). Methods for estimating the precision of survey estimates when imputation hasbeen used. Survey Methodology, 18(2):241-252.

Shabbir, J. and Gupta, S. (2005). Improved ratio estimators in stratified sampling. American Journal of Mathematical and Management Sciences, 25(3-4):293-311.

Singh, R., Chauhan, P., Sawan, N., and Smarandache, F. (2009). Improvement in estimating the 
population mean using exponential estimator in simple random sampling. International Journal of Statistics \& Economics, 3(A09):13-18.

Singh, S. (2003). Advanced Sampling Theory with Applications: How Michael 'Selected' Amy, Volume 1. Springer Science \& Business Media.

Srivastava, S. and Jhajj, H. (1983). A class of estimators of the population mean using multiauxiliary information. Calcutta Statistical Association, 32(1-2):47-56.

Upadhyaya, L. N. and Singh, H. P. (1999). Use of transformed auxiliary variable in estimating the finite population mean. Biometrical Journal, 41(5):627-636. 